\title{
Wave-Breaking Phenomena and Existence of Peakons for a Generalized Compressible Elastic-Rod Equation
}

\author{
Xiaolian Ai, ${ }^{1}$ Lingyu Jiang, ${ }^{2}$ and Ting $\mathrm{Yi}^{3}$ \\ ${ }^{1}$ Department of Mathematics, Northwest University, Xian 710069, China \\ ${ }^{2}$ Department of Mathematics, Central University of Finance and Economics, Beijing 100081, China \\ ${ }^{3}$ Department of Mathematics and Center for Nonlinear Studies, Northwest University, Xian 710069, China
}

Correspondence should be addressed to Xiaolian Ai; aixl@nwu.edu.cn

Received 30 October 2013; Revised 19 January 2014; Accepted 20 January 2014; Published 26 February 2014

Academic Editor: Ziemowit Popowicz

Copyright (C) 2014 Xiaolian Ai et al. This is an open access article distributed under the Creative Commons Attribution License, which permits unrestricted use, distribution, and reproduction in any medium, provided the original work is properly cited.

Consideration in this paper is the Cauchy problem of a generalized hyperelastic-rod wave equation. We first derive a wave-breaking mechanism for strong solutions, which occurs in finite time for certain initial profiles. In addition, we determine the existence of some new peaked solitary wave solutions.

\section{Introduction}

Consideration herein is the following generalized hyperelastic-rod wave equation:

$$
\begin{gathered}
u_{t}-u_{t x x}+\left(\frac{g(u)}{2}\right)_{x}=\gamma\left(2 u_{x} u_{x x}+u u_{x x x}\right), \\
t>0, \quad x \in \mathbb{R}, \\
u(0, x)=u_{0}(x), \quad x \in \mathbb{R},
\end{gathered}
$$

where $g: \mathbb{R} \rightarrow \mathbb{R}$ is a given $C^{\infty}$-function and the real number $\gamma$ with $\gamma \neq 0$ is a given parameter. In general, the constant $\gamma$ is given in terms of the material constants and the prestress of the rod. In fact, the authors [1,2] studied the special compressible materials which lead to values of $\gamma$ ranging from -29.4760 to 3.4174 .

When $\gamma=1, g(u)=3 u^{2}$, (1) becomes the classical Camassa-Holm $(\mathrm{CH})$ equation [3]

$$
u_{t}-u_{x x t}+3 u u_{x}+k u_{x}=2 u_{x} u_{x x}+u u_{x x x}
$$

with initial data $u(0, x)=u_{0}(x)$. While for $\gamma=0$ and $g(u)=$ $3 u^{2}$, (1) becomes the Benjamin-Bona-Mahony equation [4]. And when $g(u)=3 u^{2}, \gamma \in(-29.4760,3.4174)$, (1) is so-called the hyperelastic-rod wave equation [2].
The classical $\mathrm{CH}$ equation (2) was originally proposed as a model for surface waves [3] and has been studied extensively in the last twenty years because of its many remarkable properties: infinity of conservation laws and complete integrability [3], existence of peaked solitons and multipeakons $[3,5,6]$ (with $k=0$ ), well-posedness and breaking waves, and meaning solutions that remain bounded, while its slope becomes unbounded in finite time [7, 8]. On the other hand, if $k=0$, the $\mathrm{CH}$ equation (2) admits peaked solitary wave solutions (called peakons) which possess the form $u(t, x)=c e^{-|x-c t|}$ with speed $c \in \mathbb{R}, c \neq 0$, and their stability was studied in [9]. Recently, Gui et al. [10] proved that there exist some peaked functions which are global weak solutions to a modified Camassa-Holm equation. We should mention that the solutions to the $\mathrm{CH}$ equation (2) can be uniquely continued after wave-breaking as either a global conservative or global dissipative weak solution [11-14]. It is worth pointing out that there exists a global-in-time weak solution to the $\mathrm{CH}$ equation in the energy space [15].

For $\gamma \in \mathbb{R} \backslash\{0\}, g(u)=2 k u+(3 / 2) u^{2},(1)$ serves as a model equation for mechanical vibrations in a hyperelastic rod $[1,2]$. Similar to $\mathrm{CH}$ equation, stability of solitary wave solutions has been studied in [16]. In addition, the solutions to the hyperelastic-rod wave equation can be uniquely continued after wave breaking as a global conservative weak solution 
[17]. Moreover, there exists a global-in-time weak solution to the hyperelastic-rod wave equation in the energy space [18].

Motivated by the approaches in $[9,10]$, our goals in this paper are concerned with the wave-breaking phenomena and the existence of some new peakons of (1) with $g(u)=a u^{2}+$ $2 k u(a, k \in \mathbb{R}, a>0), \quad \gamma \neq 0$. In this case, (1) may be read as

$$
\begin{gathered}
u_{t}-u_{t x x}+\left(\frac{a}{2} u^{2}\right)_{x}+k u_{x}=\gamma\left(2 u_{x} u_{x x}+u u_{x x x}\right), \\
t>0, \quad x \in \mathbb{R}, \\
u(0, x)=u_{0}(x), \quad x \in \mathbb{R} .
\end{gathered}
$$

Introducing the momentum $m:=u-u_{x x}$, we get from (3) that

$$
\begin{array}{r}
m_{t}+\gamma u m_{x}+2 \gamma u_{x} m+(a-3 \gamma) u u_{x}+k u_{x}=0, \\
t>0, \quad x \in \mathbb{R}, \\
m(0, x)=m_{0}(x):=u_{0}-u_{0 x x}, \quad x \in \mathbb{R} .
\end{array}
$$

Note that if $p(x):=(1 / 2) e^{-|x|}, x \in \mathbb{R}$, then $\left(1-\partial_{x}^{2}\right)^{-1} f=p * f$ for all $f \in L^{2}(\mathbb{R})$ and $p *\left(u-u_{x x}\right)=u$, where $*$ denotes convolution with respect to the spatial variable $x$. Therefore, (3) can also be rewritten as the following equivalent form

$$
\begin{gathered}
u_{t}+\gamma u u_{x}=-\partial_{x} p *\left(\frac{a-\gamma}{2} u^{2}+\frac{\gamma}{2} u_{x}^{2}+k u\right), \\
t>0, \quad x \in \mathbb{R}, \\
u(0, x)=u_{0}(x), \quad x \in \mathbb{R} .
\end{gathered}
$$

We are now in a position to give the notions of strong and weak solutions.

Definition 1. If $u \in \mathscr{C}\left([0, T] ; H^{s}\right) \cap \mathscr{C}^{1}\left([0, T] ; H^{s-1}\right)$ with $s>$ $3 / 2$ and some $T>0$ satisfies (5), then $u$ is called a strong solution on $[0, T]$. If $u$ is a strong solution on $[0, T]$ for every $T>0$, then it is called a global strong solution.

Definition 2. Given initial data $u_{0} \in H^{1}(\mathbb{R})$, the function $u \in L_{\text {loc }}^{\infty}\left([0, T) ; H_{\text {loc }}^{1}(\mathbb{R})\right)$ is said to be a weak solution to the initial-value problem (5) if it satisfies the following identity:

$$
\begin{aligned}
& \int_{0}^{T} \int_{\mathbb{R}}\left[u \varphi_{t}+\frac{\gamma}{2} u^{2} \varphi_{x}\right. \\
& \left.\quad+p *\left(\frac{a-\gamma}{2} u^{2}+\frac{\gamma}{2} u_{x}^{2}+k u\right) \partial_{x} \varphi\right] d x d t \\
& \quad+\int_{\mathbb{R}} u_{0}(x) \varphi(0, x) d x=0,
\end{aligned}
$$

for any smooth test function $\varphi(t, x) \in C_{c}^{\infty}([0, T) \times \mathbb{R})$. If $u$ is a weak solution on $[0, T)$ for every $T>0$, then it is called a global weak solution.

Our main results of the present paper are Theorem 9 (wave breaking) and Theorem 10 (existence of peakons).

The remainder of the paper is organized as follows. In Section 2, the results of blow-up to strong solutions are presented in detail. It is shown that the solutions of (3) can only have singularities which correspond to wave breaking (Theorems 9). In Section 3, the existence of some new peaked solutions of (3) is verified. From this, we know that there exist some peaked solitary wave solutions for the case $k \neq 0$ (compared to the case in the Camassa-Holm equation; see Remark 11).

Notation 1. As above and henceforth, we denote the norm of the Lebesgue space $L^{p}(\mathbb{R})(1 \leq p \leq \infty)$ by $\|\cdot\|_{L^{p}}$ and the norm of the Sobolev space $H^{s}(\mathbb{R})(s \in \mathbb{R})$ by $\|\cdot\|_{H^{s}}$. We denote by $*$ the spatial convolution on $\mathbb{R}$.

\section{Wave-Breaking Phenomena}

By using the Kato's method [19], we may easily get the following results about the local well-posedness and blowup criterion of strong solutions to (5), of which proofs are similar to the one as in the $\mathrm{CH}$ equation in [7] (up to a slight modification) and we omit it here.

Theorem 3. Let $u_{0} \in H^{s}(\mathbb{R})$ with $s>3 / 2$. Then there exists a time $T>0$ such that the initial-value problem (5) has a unique strong solution $u \in \mathscr{C}\left([0, T] ; H^{s}\right) \cap \mathscr{C}^{1}\left([0, T] ; H^{s-1}\right)$ and the map $u_{0} \mapsto u$ is continuous from a neighborhood of $u_{0}$ in $H^{s}$ into $\mathscr{C}\left([0, T] ; H^{s}\right) \cap \mathscr{C}^{1}\left([0, T] ; H^{s-1}\right)$.

We are now in a position to state a blow-up criterion for (5).

Theorem 4. Let $u_{0} \in H^{s}(\mathbb{R})$ be as in Theorem 3 with $s>3 / 2$. Let $u$ be the corresponding solution to (5). Assume that $T_{u_{0}}^{*}>0$ is the maximum time of existence. Then

$$
T_{u_{0}}^{*}<\infty \Longrightarrow \int_{0}^{T_{u_{0}}^{*}}\left\|u_{x}(\tau)\right\|_{L^{\infty}} d \tau=\infty
$$

Remark 5. The blow-up criterion (7) implies that the lifespan $T_{u_{0}}^{*}$ does not depend on the regularity index $s$ of the initial data $u_{0}$. Indeed, let $u_{0}$ be in $H^{s}$ for some $s>3 / 2$ and consider some $s^{\prime} \in(3 / 2, s)$. Denote by $u_{s}$ (resp., $\left.u_{s^{\prime}}\right)$ the corresponding maximal $H^{s}$ (resp., $H^{s^{\prime}}$ ) solution given by the above theorem. Denote by $T_{s}^{*}$ (resp., $T_{s^{\prime}}^{*}$ ) the lifespan of $u_{s}$ (resp., $u_{s^{\prime}}$ ). Since $H^{s} \hookrightarrow H^{s^{\prime}}$, uniqueness ensures that $T_{s}^{*} \leq T_{s^{\prime}}^{*}$ and that $u_{s} \equiv u_{s^{\prime}}$ on $\left[0, T_{s}^{*}\right)$. Now, if $T_{s}^{*}<T_{s^{\prime}}^{*}$, then we must have $u_{s^{\prime}}$ in $\mathscr{C}\left(\left[0, T_{s}^{*}\right] ; H^{s^{\prime}}\right)$. Hence, $\partial_{x} u_{s^{\prime}} \in L^{1}\left(\left[0, T_{s}^{*}\right] ; L^{\infty}\right)$, which contradicts the above blow-up criterion (7). Therefore, $T_{s}^{*}=$ $T_{s^{\prime}}^{*}$.

Remark 6. For a strong solution $u$ in Theorem 3, the Hamiltonian functionals are conserved; that is

$$
\begin{gathered}
\frac{d}{d t} \int_{\mathbb{R}}\left(u^{2}+u_{x}^{2}\right) d x=0, \\
\frac{d}{d t} \int_{\mathbb{R}}\left(\frac{a}{3} u^{3}+\gamma u u_{x}^{2}+k u^{2}\right) d x=0,
\end{gathered}
$$

for all $t \in[0, T)$. 
The following blow-up criterion shows that the wavebreaking depends only on the infimum of $\gamma u_{x}$.

Theorem 7. Let $u_{0} \in H^{s}(\mathbb{R})$ be as in Theorem 3 with $s>3 / 2$. Then the corresponding solution $u$ to (5) blows up in finite time $T_{u_{0}}^{*}>0$ if and only if

$$
\liminf _{t \uparrow T_{u_{0}}^{*}} \inf _{x \in \mathbb{R}}\left\{\gamma u_{x}(t, x)\right\}=-\infty
$$

Proof. Since, in view of Remark 5, the existence time $T_{u_{0}}^{*}$ is independent of the choice of $s$, we need only to consider the case $s=3$, which relies on a simple density argument.

Multiplying (3) by $m$ and integrating over $\mathbb{R}$ with respect to $x$ and then integration by parts produce

$$
\begin{aligned}
\frac{1}{2} \frac{d}{d t} \int_{\mathbb{R}} m^{2} d x= & -\frac{a}{2} \int_{\mathbb{R}}\left(u^{2}\right)_{x} m d x \\
& +\gamma \int_{\mathbb{R}} m\left(2 u_{x} u_{x x}+u u_{x x x}\right) d x \\
= & -a \int_{\mathbb{R}} u u_{x} m d x+2 \gamma \int_{\mathbb{R}} u_{x}(u-m) m d x \\
& +\gamma \int_{\mathbb{R}} u\left(u_{x}-m_{x}\right) m d x \\
= & (3 \gamma-a) \int_{\mathbb{R}} u u_{x} m d x-\frac{3}{2} \gamma \int_{\mathbb{R}} u_{x} m^{2} d x .
\end{aligned}
$$

We next differentiate (3) with respect to $x$ to get

$$
\begin{aligned}
m_{x t}= & (3 \gamma-a)\left(u_{x}^{2}+u^{2}-u m\right) \\
& -3 \gamma u_{x} m_{x}-2 \gamma u m-\gamma u m_{x x}+k(m-u) .
\end{aligned}
$$

Multiplying by $m_{x}$ then integrating over $\mathbb{R}$ with respect to $x$ lead to

$$
\begin{aligned}
\frac{1}{2} \frac{d}{d t} \int_{\mathbb{R}} m_{x}^{2} d x \\
=-2(3 \gamma-a) \int_{\mathbb{R}} u_{x}(u-m) m d x \\
\quad-2(3 \gamma-a) \int_{\mathbb{R}} u_{x} u m d x \\
\quad-(3 \gamma-a) \int_{\mathbb{R}} u m m_{x} d x-3 \gamma \int_{\mathbb{R}} u_{x} m_{x}^{2} d x \\
\quad-2 \gamma \int_{\mathbb{R}} u m m_{x} d x+\frac{\gamma}{2} \int_{\mathbb{R}} u_{x} m_{x}^{2} d x \\
=-4(3 \gamma-a) \int_{\mathbb{R}} u_{x} u m d x \\
+(5 a-17 \gamma) \int_{\mathbb{R}} u m m_{x} d x-\frac{5}{2} \gamma \int_{\mathbb{R}} u_{x} m_{x}^{2} d x .
\end{aligned}
$$

Therefore,

$$
\begin{aligned}
& \frac{d}{d t} \int_{\mathbb{R}}\left(m^{2}+m_{x}^{2}\right) d x \\
& =-6(3 \gamma-a) \int_{\mathbb{R}} u_{x} u m d x+2(5 a-17 \gamma) \int_{\mathbb{R}} u m m_{x} d x \\
& \quad-\int_{\mathbb{R}}\left(\gamma u_{x}\right)\left(3 m^{2}+5 m_{x}^{2}\right) d x
\end{aligned}
$$

If $\gamma u_{x}$ is bounded from below on $\left[0, T_{m_{0}}^{*}\right) \times \mathbb{R}$, that is, there exists a positive constant $C_{1}>0$ such that $\gamma u_{x} \geq-C_{1}$ on $\left[0, T_{m_{0}}^{*}\right) \times \mathbb{R}$, then the above estimate implies

$$
\begin{aligned}
& \frac{d}{d t} \int_{\mathbb{R}}\left(m^{2}+m_{x}^{2}\right) d x \\
& \leq 5 C_{1} \int_{\mathbb{R}}\left(m^{2}+m_{x}^{2}\right) d x \\
& \quad+|2(3 \gamma-a)|\|u(t, \cdot)\|_{L^{\infty}} \int_{\mathbb{R}}\left(u_{x}^{2}+m^{2}\right) d x \\
& \quad+|5 a-17 \gamma|\|u(t, \cdot)\|_{L^{\infty}} \int_{\mathbb{R}}\left(m^{2}+m_{x}^{2}\right) d x \\
& \leq C_{2}\left(a, \gamma,\left\|u_{0}\right\|_{H^{1}}\right) \int_{\mathbb{R}}\left(m^{2}+m_{x}^{2}\right) d x,
\end{aligned}
$$

where we used (8) so that $\|u(t, \cdot)\|_{L^{\infty}}^{2} \leq(1 / 2) E(u(t, \cdot))=(1 / 2)$ $E\left(u_{0}\right)=(1 / 2)\left\|u_{0}\right\|_{H^{1}}^{2}$. Applying Gronwall's inequality then yields

$$
\|m(t)\|_{H^{1}}^{2}=\int_{\mathbb{R}}\left(m^{2}+m_{x}^{2}\right) d x \leq e^{C_{2} t}\left\|m_{0}\right\|_{H^{1}}^{2}
$$

for $t \in\left[0, T_{m_{0}}^{*}\right)$, which ensures that the solution $m(t, x)$ does not blow up in finite time.

On the other hand, if

$$
\liminf _{t \uparrow T_{u_{0}}^{*}}\left[\inf _{x \in \mathbb{R}}\left(\gamma u_{x}(t, x)\right)\right]=-\infty,
$$

by Theorem 3 for the existence of local strong solutions and the Sobolev embedding theorem, we infer that the solution will blow up in finite time. The proof of Theorem 7 is then complete.

For $\gamma \neq 0$, we define

$$
\mathfrak{S}(t) \stackrel{\text { def }}{=} \operatorname{sign}\{\gamma\} \inf _{x \in \mathbb{R}}\left[u_{x}(t, x) \operatorname{sign}\{\gamma\}\right], \quad t \geq 0,
$$

where $\operatorname{sign}(\gamma)$ is the sign function of $\gamma \in \mathbb{R}$, and set $\mathfrak{g}_{0}:=\mathfrak{g}(0)$. Then, thanks to Theorem 3 , for every $t \in[0, T)$ there exists at least one point $\xi(t) \in \mathbb{R}$ with $\mathfrak{g}(t)=u_{x}(t, \xi(t))$. Just as the proof given in $[8]$, one can show the following property of $\mathfrak{S}(t)$.

Lemma 8. Let $u(t)$ be the solution to (5) on $[0, T)$ with initial data $u_{0} \in H^{2}(\mathbb{R})$, as given by Theorem 3 . Then the function $\mathfrak{S}(t)$ is almost everywhere differentiable on $[0, T)$, with

$$
\frac{d \mathfrak{g}}{d t}=u_{t x}(t, \xi(t)) \quad \text { a.e. on }(0, T) \text {. }
$$


With Lemma 8 in hand, we may get the following result about the wave-breaking phenomena of solutions to the generalized hyperelastic-rod wave equation (3).

Theorem 9. Let $a>0, \gamma \neq 0$, and $u_{0} \in H^{s}(\mathbb{R})$ with $s>3 / 2$. Assume that

$$
\begin{gathered}
\inf _{x \in \mathbb{R}}\left\{u_{0}^{\prime}(x)\right\}<-K \quad \text { if } \gamma>0, \\
\text { or } \quad \sup _{x \in \mathbb{R}}\left\{u_{0}^{\prime}(x)\right\}>K \quad \text { if } \gamma<0,
\end{gathered}
$$

where

$$
K:= \begin{cases}\sqrt{\frac{|a-\gamma|}{2|\gamma|}\left\|u_{0}\right\|_{H^{1}},} & \text { if } \gamma \in(-\infty, 0) \cup(a,+\infty), \\ \sqrt{\frac{a-\left(1+\alpha_{0}\right) \gamma}{2 \gamma}\left\|u_{0}\right\|_{H^{1}},} & \text { if } \gamma \in\left(0, \frac{a}{1+\alpha_{0}}\right], \\ 0, & \text { if } \gamma \in\left(\frac{a}{1+\alpha_{0}}, a\right)\end{cases}
$$

with $\alpha_{0}=\sqrt{(4 a-3 \gamma) / 4 \gamma}-(1 / 2)$ for $\gamma \in(0, a)$. Then the corresponding solution to (5) blows up in finite time. Moreover, the maximal time of existence is estimated above by

$$
0<T \leq \frac{-2 \inf _{x \in \mathbb{R}}\left\{\operatorname{sign}(\gamma) u_{0}^{\prime}(x)\right\}}{|\gamma|\left[\left(\inf _{x \in \mathbb{R}}\left\{\operatorname{sign}(\gamma) u_{0}^{\prime}(x)\right\}\right)^{2}-K^{2}\right]}<+\infty .
$$

Proof. Let $T>0$ be the maximal existence time of the solution $u(t, x)$ to (5) with initial data $u_{0}$ as stated in Theorem 3. Applying Theorem 3, Remark 5, and a simple density argument, we need only to show that Theorem 9 holds with some $s \geq 5$. Here we assume $s=5$ to prove the theorem.

Firstly, thanks to Lemma 8 and the definition of $\mathfrak{g}(t)$, there are $x_{0} \in \mathbb{R}$ and $\xi(t) \in \mathbb{R}$ such that $u_{0}^{\prime}\left(x_{0}\right)=\inf _{x \in \mathbb{R}} u_{0}^{\prime}(x)$, (18) holds, and

$$
u_{x x}(t, \xi(t))=0, \quad \forall t \in[0, T)
$$

Differentiating (5) with respect to $x$ and using the identity $\partial_{x}^{2} p * f=p * f-f$, we obtain

$$
\begin{aligned}
u_{x t}= & -\frac{\gamma}{2} u_{x}^{2}-\gamma u u_{x x}+\frac{a-\gamma}{2} u^{2} \\
& -p *\left(\frac{a-\gamma}{2} u^{2}+\frac{\gamma}{2} u_{x}^{2}\right)-k u_{x x},
\end{aligned}
$$

which along with (22) implies

$$
\begin{aligned}
\frac{d}{d t} \mathfrak{G}(t)= & -\frac{\gamma}{2} \mathfrak{g}(t)^{2}+\frac{a-\gamma}{2} u^{2}(t, \xi(t)) \\
& -p *\left(\frac{a-\gamma}{2} u^{2}+\frac{\gamma}{2} u_{x}^{2}\right)(t, \xi(t)) \quad \text { a.e. on }(0, T)
\end{aligned}
$$

For the case $\gamma \geq a$, thanks to the Young's inequality and the Sobolev inequality, we get from Remark 6 that, for all $t \in$ $[0, T)$,

$$
\begin{aligned}
\left\|p * \frac{a-\gamma}{2} u^{2}\right\|_{L^{\infty}} & \leq \frac{\gamma-a}{2}\|u(t)\|_{L^{\infty}}^{2} \\
& \leq \frac{\gamma-a}{4}\|u(t)\|_{H^{1}}^{2}=\frac{\gamma-a}{4}\left\|u_{0}\right\|_{H^{1}}^{2} .
\end{aligned}
$$

Note that $p *\left((\gamma / 2) u_{x}^{2}\right) \geq 0$ and $((a-\gamma) / 2) u^{2}(t, \xi(t)) \leq 0$, we infer that

$$
\frac{d}{d t} \mathfrak{g}(t) \leq-\frac{\gamma}{2} \mathfrak{g}^{2}(t)+\frac{\gamma-a}{4}\left\|u_{0}\right\|_{H^{1}}^{2} \quad \text { a.e. on }(0, T) \text {. }
$$

Set $K:=\sqrt{(\gamma-a) / 2 \gamma}\left\|u_{0}\right\|_{H^{1}}$; we have

$$
\frac{d}{d t} \mathfrak{g}(t) \leq-\frac{\gamma}{2} \mathfrak{g}^{2}(t)+\frac{\gamma}{2} K^{2} \quad \text { a.e. on }(0, T)
$$

We now claim that if $\mathfrak{g}(0)<-K$, then $\mathfrak{g}(t)<-K$ for all $t \in(0, T)$.

In fact, assuming that the contrary would, in view of $\mathfrak{S}(t)(t)$ being continuous, ensure the existence of some $t_{0} \in$ $(0, T)$, such that $\mathfrak{g}(t)<-K$ in $\left(0, t_{0}\right)$ and $\mathfrak{g}\left(t_{0}\right)=-K$. Combining this with $(27)$ would give $(d / d t) \mathfrak{g}(t)<0$ a.e. on $\left[0, t_{0}\right)$. Since $\mathfrak{G}(t)$ is absolutely continuous on $\left[0, t_{0}\right]$, an integration of this inequality would give the following inequality and we get the contradiction $\mathfrak{g}\left(t_{0}\right)<\mathfrak{g}(0)<-K$. This proves the previous claim.

Therefore it follows from (27) that $(d / d t) \mathfrak{g}(t)<$ 0 a.e. on $(0, T)$ which implies that $\mathfrak{g}(t)$ is strictly decreasing on $[0, T)$.

Set $\delta:=(\gamma / 2)\left(1-\left(K / u_{0}^{\prime}\left(x_{0}\right)\right)^{2}\right) \in(0, \gamma / 2)$. Then we have $K^{2} /(1-(2 / \gamma) \delta)=\left(u_{0}^{\prime}\left(x_{0}\right)\right)^{2}<\mathfrak{g}^{2}(t)$, that is, $K^{2}<(1-$ $(2 / \gamma) \delta) \mathfrak{g}^{2}(t)$. Therefore it follows that

$$
\frac{d}{d t} \mathfrak{G}(t) \leq-\frac{\gamma}{2} \mathfrak{g}^{2}(t)\left[1-\left(1-\frac{2}{\gamma} \delta\right)\right]=-\delta \mathfrak{S}^{2}(t),
$$

$$
\text { a.e. on }(0, T) \text {, }
$$

which leads to

$$
\mathfrak{G}(t) \leq \frac{u_{0}^{\prime}\left(x_{0}\right)}{1+\delta t u_{0}^{\prime}\left(x_{0}\right)} \longrightarrow-\infty, \quad \text { as } t \longrightarrow-\frac{1}{\delta u_{0}^{\prime}\left(x_{0}\right)}
$$

This implies that the maximal time of existence $T$ satisfies

$$
T \leq-\frac{1}{\delta u_{0}^{\prime}\left(x_{0}\right)}=\frac{2 \inf _{x \in \mathbb{R}} u_{0}^{\prime}(x)}{\gamma\left[K^{2}-\left(\inf _{x \in \mathbb{R}} u_{0}^{\prime}(x)\right)^{2}\right]}<+\infty
$$

In consequence, we have

$$
\liminf _{t \uparrow T}\left(\inf _{x \in \mathbb{R}} \gamma u_{x}(t, x)\right)=-\infty
$$

which along with Theorem 7 applied completes the proof of Theorem 9 for the case $\gamma \geq a$. 
Next, to deal with the case $0<\gamma<a$, we need first to verify the following inequality for any positive real number $\alpha$ :

$$
p *\left(\left(\alpha^{2}+\alpha\right) u^{2}+u_{x}^{2}\right)(x) \geq \alpha u^{2}(x) \quad \text { for } \forall u \in H^{3}(\mathbb{R})
$$

Indeed, observe that the inequality

$$
\begin{aligned}
& e^{-x} \int_{-\infty}^{x} e^{y}\left(\alpha^{2} u^{2}+u_{x}^{2}\right)(y) d y \\
& \geq 2 \alpha e^{-x} \int_{-\infty}^{x} e^{y} u(y) u_{x}(y) d y \\
& =\alpha e^{-x} \int_{-\infty}^{x} e^{y} \frac{d}{d y}\left(u^{2}(y)\right) d y \\
& =\alpha u^{2}(x)-\alpha e^{-x} \int_{-\infty}^{x} e^{y} u^{2}(y) d y
\end{aligned}
$$

yields

$$
e^{-x} \int_{-\infty}^{x} e^{y}\left(\left(\alpha^{2}+\alpha\right) u^{2}+u_{x}^{2}\right)(y) d y \geq \alpha u^{2}(x)
$$

Whereas the inequality

$$
\begin{aligned}
e^{x} \int_{x}^{\infty} & e^{-y}\left(\alpha^{2} u^{2}+u_{x}^{2}\right)(y) d y \\
& \geq-2 \alpha e^{x} \int_{x}^{\infty} e^{-y} u(y) u_{x}(y) d y \\
& =-\alpha e^{x} \int_{x}^{\infty} e^{-y} \frac{d}{d y}\left(u^{2}(y)\right) d y \\
& =\alpha u^{2}(x)-\alpha e^{x} \int_{x}^{\infty} e^{-y} u^{2}(y) d y
\end{aligned}
$$

leads to

$$
e^{x} \int_{x}^{\infty} e^{-y}\left(\left(\alpha^{2}+\alpha\right) u^{2}+u_{x}^{2}\right)(y) d y \geq \alpha u^{2}(x),
$$

which along with (34) gives rise to (32).

Notice that $\alpha_{0}=\sqrt{(4 a-3 \gamma) / 4 \gamma}-(1 / 2)$ is the only positive root of $\alpha^{2}+\alpha=(a-\gamma) / \gamma$; we get from (32) that

$$
p *\left(\frac{a-\gamma}{2} u^{2}+\frac{\gamma}{2} u_{x}^{2}\right)(t, \xi(t)) \geq \frac{\gamma \alpha_{0}}{2} u_{x}^{2}(t, \xi(t))
$$

which together with (24) implies that

$$
\begin{aligned}
\frac{d}{d t} \mathfrak{g}(t) \leq & -\frac{\gamma}{2} \mathfrak{g}(t)^{2} \\
& +\frac{a-\left(1+\alpha_{0}\right) \gamma}{2} u^{2}(t, \xi(t)) \quad \text { a.e. on }(0, T)
\end{aligned}
$$

Thanks to the Sobolev inequality and Remark 6, we get that, for any $t \in(0, T),\|u(t)\|_{L^{\infty}}^{2} \leq(1 / 2)\|u(t)\|_{H^{1}}^{2}=(1 / 2)\left\|u_{0}\right\|_{H^{1}}^{2}$, which along with (38) implies that (27) holds for

$$
K:= \begin{cases}\sqrt{\frac{a-\left(1+\alpha_{0}\right) \gamma}{2 \gamma}}\left\|u_{0}\right\|_{H^{1}}, & \text { if } \gamma \in\left(0, \frac{a}{1+\alpha_{0}}\right], \\ 0, & \text { if } \gamma \in\left(\frac{a}{1+\alpha_{0}}, a\right) .\end{cases}
$$

Repeating the above argument in the proof of (31) for the case $\gamma \geq a$ leads to (31), and then the proof of Theorem 9 is complete for the case $0<\gamma<a$.

Let us now consider the case $\gamma<0$. It follows from (24) that

$$
\frac{d}{d t} \mathfrak{G}(t) \geq \frac{|\gamma|}{2} \mathfrak{g}^{2}(t)-\frac{a-\gamma}{4}\left\|u_{0}\right\|_{H^{1}}^{2} \quad \text { a.e. on }(0, T),
$$

where we used Young's inequality so that, for all $t \in[0, T)$,

$$
\left\|p * u^{2}\right\|_{L^{\infty}} \leq\|u(t)\|_{L^{\infty}}^{2} \leq \frac{1}{2}\|u(t)\|_{H^{1}}^{2} .
$$

Therefore, set $K:=\sqrt{(a-\gamma) /-2 \gamma}\left\|u_{0}\right\|_{H^{1}}$; we may get

$$
\frac{d}{d t} \mathfrak{g}(t) \geq \frac{|\gamma|}{2} \mathfrak{g}^{2}(t)-\frac{|\gamma|}{2} K^{2} \quad \text { a.e. on }(0, T)
$$

Repeating the above argument in the proof of (31) for the case $\gamma \geq a$ again ends the proof of Theorem 9 .

\section{Peaked Solitary Wave Solution}

In this section we consider the existence of peaked solitary wave solutions of (3) in the case of $g(u)=a u^{2}+2 k u(a, k \in \mathbb{R}$ and $k \neq 0)$, which can be understood as global weak solutions.

Theorem 10. For any $\gamma \in \mathbb{R} \backslash\{a / 3,0\}$, a $\gamma>0$, and $k \neq 0$, the peaked function of the form

$$
\bar{u}_{c}(t, x)=\frac{c}{\gamma} e^{-\sqrt{a / 3 \gamma}|x-c t|}, \quad \text { with } c=\frac{3 k \gamma}{3 \gamma-a}
$$

is a global weak solution to (3) in the sense of Definition (2).

Remark 11. It is known that for the Camassa-Holm equation (that is, (5) with $\gamma=1$ and $a=3$ ), for any $c \neq 0$, the peaked function of the form $u_{c}(t, x)=c e^{-|x-c t|}$ is a global weak solution to the Camassa-Holm equation with the case $k=0$ in the sense of Definition (2), and if $k \neq 0$, the Camassa-Holm equation has no peaked solution. Here Theorem 10 implies that, if $k \neq 0$, (5) still has some peaked solutions, as long as $\gamma \neq a / 3$ and 0 .

Proof of Theorem 10. The proof of the theorem is motivated by the method in [10]. First, we can reduce the result to the 
one with the case $\gamma=1$. In fact, multiplying $\gamma$ with (1) and setting $\bar{u}=\gamma u$, (1) become the following form:

$$
\begin{gathered}
\bar{u}_{t}-\bar{u}_{t x x}+\left(\frac{a}{2 \gamma} \bar{u}^{2}+k \bar{u}\right)_{x}=2 \bar{u}_{x} \bar{u}_{x x}+\bar{u} \bar{u}_{x x x}, \\
t>0, \quad x \in \mathbb{R}, \\
\bar{u}(0, x)=\bar{u}_{0}(x), \quad x \in \mathbb{R} .
\end{gathered}
$$

We claim that, for all $t \in \mathbb{R}^{+}$,

$$
\partial_{x} \bar{u}_{c}(t, x)=-\sqrt{\frac{a}{3 \gamma}} \operatorname{sign}(x-c t) \bar{u}_{c}(t, x) \in L^{\infty}(\mathbb{R}),
$$

in the sense of distribution $\mathcal{S}^{\prime}(\mathbb{R})$.

Indeed, for any test function $\varphi(\cdot) \in C_{c}^{\infty}(\mathbb{R})$, we may get by using integration by parts that

$$
\begin{aligned}
\int_{\mathbb{R}} & \operatorname{sign}(y) e^{-\sqrt{a / 3 \gamma}|y|} \varphi(y) d y \\
= & -\int_{-\infty}^{0} e^{\sqrt{a / 3 \gamma} y} \varphi(y) d y+\int_{0}^{+\infty} e^{-\sqrt{a / 3 \gamma} y} \varphi(y) d y \\
= & -\varphi(0)+\sqrt{\frac{3 \gamma}{a}} \int_{-\infty}^{0} e^{\sqrt{a / 3 \gamma} y} \varphi^{\prime}(y) d y+\varphi(0) \\
& +\sqrt{\frac{3 \gamma}{a}} \int_{0}^{+\infty} e^{-\sqrt{a / 3 \gamma} y} \varphi^{\prime}(y) d y \\
= & \sqrt{\frac{3 \gamma}{a}} \int_{\mathbb{R}} e^{-\sqrt{a / 3 \gamma}|y|} \varphi^{\prime}(y) d y
\end{aligned}
$$

which along with the fact that $\operatorname{sign}(\cdot-c t) \bar{u}_{c}(t, \cdot) \in L^{\infty}(\mathbb{R})$ ends the proof of the claim.

Let us now set $\bar{u}_{0, c}(x):=\bar{u}_{c}(0, x)$ for $x \in \mathbb{R}$. Then we have

$$
\lim _{t \rightarrow 0^{+}}\left\|\bar{u}_{c}(t, \cdot)-\bar{u}_{0, c}(\cdot)\right\|_{W^{1, \infty}}=0
$$

Similar to the proof of (46), we get that, for all $t \geq 0$,

$$
\partial_{t} \bar{u}_{c}(t, x)=c \operatorname{sign}(x-c t) \bar{u}_{c}(t, x) \in L^{\infty}(\mathbb{R}) .
$$

Therefore, from (46), (48), (49), and $c=3 k \gamma /(3 \gamma-a)$ and $\gamma=1$, together with integration by parts applied, we obtain that, for every test function $\phi(t, x) \in C_{c}^{\infty}([0,+\infty) \times \mathbb{R})$,

$$
\begin{aligned}
& \int_{0}^{+\infty} \int_{\mathbb{R}}\left(\bar{u}_{d} \partial_{t} \phi+\frac{1}{2} \bar{u}_{c}^{2} \partial_{x} \phi\right) d x d t \\
& +\int_{\mathbb{R}} \bar{u}_{c}(0, x) \phi(0) d x d t \\
& =-\int_{0}^{+\infty} \int_{\mathbb{R}} \phi\left(\partial_{t} \bar{u}_{c}+\bar{u}_{c} \partial_{x} \bar{u}_{c}\right) d x d t \\
& =-\int_{0}^{+\infty} \int_{\mathbb{R}} \phi\left(c \sqrt{\frac{a}{3}} \operatorname{sign}(x-c t) c e^{-\sqrt{a / 3}|x-c t|}\right. \\
& -\sqrt{\frac{a}{3}} \operatorname{sign}(x-c t) \\
& \left.\times c^{2} e^{-2 \sqrt{a / 3}|x-c t|}\right) d x d t \\
& =-\int_{0}^{+\infty} \int_{\mathbb{R}} \phi c^{2} \sqrt{\frac{a}{3}} \operatorname{sign}(x-c t) \\
& \times\left(e^{-\sqrt{a / 3}|x-c t|}-e^{-2 \sqrt{a / 3}|x-c t|}\right) d x d t, \\
& \int_{0}^{+\infty} \int_{\mathbb{R}} p *\left(\frac{a-1}{2} \bar{u}_{c}^{2}+\frac{1}{2}\left(\partial_{x} \bar{u}_{c}\right)^{2}+k \bar{u}_{c}\right) \phi_{x} d x d t \\
& =-\int_{0}^{+\infty} \int_{\mathbb{R}} \phi \partial_{x} p *\left(\frac{a-1}{2} \bar{u}_{c}^{2}+\frac{1}{2}\left(\partial_{x} \bar{u}_{c}\right)^{2}+k \bar{u}_{c}\right) d x d t .
\end{aligned}
$$

Notice from (46) that $\partial_{x} p=-(1 / 2) \operatorname{sign}(x) e^{-|x|}$ for $x \in \mathbb{R}$; we have

$$
\begin{array}{rl}
\partial_{x} p & *\left(\frac{a-1}{2} \bar{u}_{c}^{2}+\frac{1}{2}\left(\partial_{x} \bar{u}_{c}\right)^{2}+k \bar{u}_{c}\right) \\
=-\frac{1}{2} \int_{-\infty}^{+\infty} & \operatorname{sign}(x-y) e^{-|x-y|} \\
& \times\left(\frac{4 a-3}{6} c^{2} e^{-2 \sqrt{a / 3}|y-c t|}+k c e^{-\sqrt{a / 3}|y-c t|}\right) d y .
\end{array}
$$

When $x>c t$, we split the right hand side of (52) into the following three parts:

$$
\begin{aligned}
\partial_{x} p * & \left(\frac{a-1}{2} \bar{u}_{c}^{2}+\frac{1}{2}\left(\partial_{x} \bar{u}_{c}\right)^{2}+k \bar{u}_{c}\right) \\
=- & \frac{1}{2}\left(\int_{-\infty}^{c t}+\int_{c t}^{x}+\int_{x}^{+\infty}\right) \operatorname{sign}(x-y) e^{-|x-y|} \\
& \times\left(\frac{4 a-3}{6} c^{2} e^{-2 \sqrt{a / 3}|y-c t|}+k c e^{-\sqrt{a / 3}|y-c t|}\right) d y \\
=: & I_{1}+I_{2}+I_{3} .
\end{aligned}
$$


We directly compute $I_{1}$ as follows:

$$
\begin{aligned}
I_{1}= & -\frac{1}{2} \int_{-\infty}^{c t} e^{-(x-y)}\left(\frac{4 a-3}{6} c^{2} e^{2 \sqrt{a / 3}(y-c t)}\right. \\
& \left.+k c e^{\sqrt{a / 3}(y-c t)}\right) d y \\
= & \left(-\frac{4 a-3}{12(1+2 \sqrt{a / 3})} d^{2}-\frac{k c}{2(1+\sqrt{a / 3})}\right) e^{-(x-c t)} .
\end{aligned}
$$

Similarly, we have

$$
\begin{aligned}
I_{2}= & \left(-\frac{4 a-3}{12(1-2 \sqrt{a / 3})} c^{2}\right) e^{-2 \sqrt{a / 3}(x-c t)} \\
& +\left(-\frac{k c}{2(1-\sqrt{a / 3})}\right) e^{-\sqrt{a / 3}(x-c t)} \\
& +\left(\frac{4 a-3}{12(1-2 \sqrt{a / 3})} c^{2}+\frac{k c}{2(1-\sqrt{a / 3})}\right) e^{-(x-c t)}
\end{aligned}
$$

and $I_{3}=((4 a-3) /(12(1+2 \sqrt{a / 3}))) c^{2} e^{-2 \sqrt{a / 3}(x-c t)}+(k c /(2(1+$ $\sqrt{a / 3}))) e^{-\sqrt{a / 3}(x-c t)}$. From this, we deduce that, for $x>c t$,

$$
\begin{array}{rl}
\partial_{x} p & *\left(\frac{a-1}{2} \bar{u}_{c}^{2}+\frac{1}{2}\left(\partial_{x} \bar{u}_{c}\right)^{2}+k \bar{u}_{c}\right) \\
& =\sqrt{\frac{a}{3}} c^{2}\left(e^{-2 \sqrt{a / 3}(x-c t)}-e^{-\sqrt{a / 3}(x-c t)}\right) .
\end{array}
$$

And for the case $x \leq c t$, we spilt the right hand side of (51) into the following three parts:

$$
\begin{aligned}
\partial_{x} p *( & \left.\frac{a-1}{2} \bar{u}_{c}^{2}+\frac{1}{2}\left(\partial_{x} \bar{u}_{c}\right)^{2}+k \bar{u}_{c}\right) \\
= & -\frac{1}{2}\left(\int_{-\infty}^{x}+\int_{x}^{c t}+\int_{c t}^{+\infty}\right) \operatorname{sign}(x-y) e^{-|x-y|} \\
& \quad\left(\frac{4 a-3}{6} c^{2} e^{-2 \sqrt{a / 3}|y-c t|}+k c e^{-\sqrt{a / 3}|y-c t|}\right) d y \\
= & I I_{1}+I I_{2}+I I_{3} .
\end{aligned}
$$

We compute

$$
\begin{aligned}
I_{1}= & -\frac{1}{2} \int_{-\infty}^{x} e^{-(x-y)}\left(\frac{4 a-3}{6} c^{2} e^{2 \sqrt{a / 3}(y-c t)}\right. \\
& \left.+k c e^{\sqrt{a / 3}(y-c t)}\right) d y \\
= & -\frac{4 a-3}{12(1+2 \sqrt{a / 3})} c^{2} e^{2 \sqrt{a / 3}(x-c t)} \\
& -\frac{k c}{2(1+\sqrt{a / 3})} e^{\sqrt{a / 3}(x-c t)} .
\end{aligned}
$$

Similarly, we have

$$
\begin{aligned}
I_{2}= & \frac{4 a-3}{12(2 \sqrt{a / 3}-1)} c^{2}\left(e^{x-c t}-e^{2 \sqrt{a / 3}(x-c t)}\right) \\
& +\frac{k c}{2(\sqrt{a / 3}-1)}\left(e^{x-c t}-e^{\sqrt{a / 3}(x-c t)}\right)
\end{aligned}
$$

and $I_{3}=\left(((4 a-3) /(12(1+2 \sqrt{a / 3}))) c^{2}+k c /(2(1+\right.$ $\sqrt{a / 3}))) e^{x-c t}$, which along with (57) and (58) implies that, for $x \leq c t$,

$$
\begin{array}{rl}
\partial_{x} p & *\left(\frac{a-1}{2} \bar{u}_{c}^{2}+\frac{1}{2}\left(\partial_{x} \bar{u}_{c}\right)^{2}+k \bar{u}_{c}\right) \\
& =\sqrt{\frac{a}{3}} c^{2}\left(e^{\sqrt{a / 3}(x-c t)}-e^{2 \sqrt{a / 3}(x-c t)}\right) .
\end{array}
$$

From (56) and (60), we obtain

$$
\begin{aligned}
& \int_{0}^{+\infty} \int_{\mathbb{R}} p *\left(\frac{a-1}{2} \bar{u}_{c}^{2}+\frac{1}{2}\left(\partial_{x} \bar{u}_{c}\right)^{2}+k \bar{u}_{c}\right) \phi_{x} d x d t \\
& =\left\{\begin{array}{l}
-\sqrt{\frac{a}{3}} c^{2} \\
\times \int_{0}^{+\infty} \int_{\mathbb{R}} \phi\left(e^{-2 \sqrt{a / 3}(x-c t)}\right. \\
\left.-\sqrt{\frac{a}{3}} c^{2}-e^{-\sqrt{a / 3}(x-c t)}\right) d x d t, \quad x>c t, \\
\times \int_{0}^{+\infty} \int_{\mathbb{R}} \phi\left(e^{\sqrt{a / 3}(x-c t)}\right. \\
\left.-e^{2 \sqrt{a / 3}(x-c t)}\right) d x d t, \quad x \leq c t .
\end{array}\right.
\end{aligned}
$$

On the other hand, by using the form of $\bar{u}_{c}$, we get from (50) that

$$
\begin{aligned}
& \int_{0}^{+\infty} \int_{\mathbb{R}}\left(\bar{u}_{c} \partial_{t} \phi+\frac{1}{2} \bar{u}_{c}^{2} \partial_{x} \phi\right) d x d t \\
& \quad+\int_{\mathbb{R}} \bar{u}_{d}(0, x) \phi(0) d x d t \\
& =\left\{\begin{array}{l}
-\sqrt{\frac{a}{3} c^{2}} \\
\times \int_{0}^{+\infty} \int_{\mathbb{R}} \phi\left(e^{-\sqrt{a / 3}(x-c t)}-e^{-2 \sqrt{a / 3}(x-c t)}\right) d x d t, \quad x>c t, \\
\sqrt{\frac{a}{3}} c^{2} \\
\times \int_{0}^{+\infty} \int_{\mathbb{R}} \phi\left(e^{\sqrt{a / 3}(x-c t)}\right. \\
\left.-e^{2 \sqrt{a / 3}(x-c t)}\right) d x d t, \quad x \leq c t .
\end{array}\right.
\end{aligned}
$$


Therefore, for every test function $\phi(t, x) \in C_{c}^{\infty}([0,+\infty) \times \mathbb{R})$, we conclude that

$$
\begin{aligned}
& \int_{0}^{+\infty} \int_{\mathbb{R}}\left[\bar{u}_{c} \partial_{t} \phi+\frac{1}{2} \bar{u}_{c}^{2} \partial_{x} \phi\right. \\
& \left.\quad+p *\left(\frac{a-1}{2} \bar{u}_{c}^{2}+\frac{1}{2}\left(\partial_{x} \bar{u}_{c}\right)^{2}+k \bar{u}_{c}\right) \partial_{x} \phi\right] d x d t \\
& \quad+\int_{\mathbb{R}} \bar{u}_{c}(0, x) \phi(0, x) d x=0,
\end{aligned}
$$

which completes the proof of Theorem 10.

\section{Conflict of Interests}

The authors declare that there is no conflict of interests regarding the publication of this paper.

\section{Acknowledgments}

The work of Ai is partially supported by the NSF of China under Grant 11001111. The authors would like to thank the referees for constructive suggestions and comments.

\section{References}

[1] H.-H. Dai, "Model equations for nonlinear dispersive waves in a compressible Mooney-Rivlin rod," Acta Mechanica, vol. 127, no. 1-4, pp. 193-207, 1998.

[2] H.-H. Dai and Y. Huo, "Solitary shock waves and other travelling waves in a general compressible hyperelastic rod," Proceedings of the Royal Society of London A, vol. 456, no. 1994, pp. 331-363, 2000.

[3] R. Camassa and D. D. Holm, "An integrable shallow water equation with peaked solitons," Physical Review Letters, vol. 71, no. 11, pp. 1661-1664, 1993.

[4] T. B. Benjamin, J. L. Bona, and J. J. Mahony, "Model equations for long waves in nonlinear dispersive systems," Philosophical Transactions of the Royal Society of London A, vol. 272, no. 1220, pp. 47-78, 1972.

[5] M. S. Alber, R. Camassa, D. D. Holm, and J. E. Marsden, "The geometry of peaked solitons and billiard solutions of a class of integrable PDEs," Letters in Mathematical Physics, vol. 32, no. 2, pp. 137-151, 1994.

[6] C. Cao, D. D. Holm, and E. S. Titi, “Traveling wave solutions for a class of one-dimensional nonlinear shallow water wave models," Journal of Dynamics and Differential Equations, vol. 16, no. 1, pp. 167-178, 2004.

[7] A. Constantin and J. Escher, "Global existence and blow-up for a shallow water equation," Annali della Scuola Normale Superiore di Pisa. Classe di Scienze, vol. 26, no. 2, pp. 303-328, 1998.

[8] A. Constantin and J. Escher, "Wave breaking for nonlinear nonlocal shallow water equations," Acta Mathematica, vol. 181, no. 2, pp. 229-243, 1998.

[9] A. Constantin and W. A. Strauss, "Stability of peakons," Communications on Pure and Applied Mathematics, vol. 53, no. 5, pp. 603-610, 2000.

[10] G. Gui, Y. Liu, P. J. Olver, and C. Qu, "Wave-breaking and peakons for a modified Camassa-Holm equation," Communications in Mathematical Physics, vol. 319, no. 3, pp. 731-759, 2013.
[11] A. Bressan and A. Constantin, "Global conservative solutions of the Camassa-Holm equation," Archive for Rational Mechanics and Analysis, vol. 183, no. 2, pp. 215-239, 2007.

[12] A. Bressan and A. Constantin, "Global dissipative solutions of the Camassa-Holm equation," Analysis and Applications, vol. 5, no. 1, pp. 1-27, 2007.

[13] H. Holden and X. Raynaud, "Global conservative solutions of the Camassa-Holm equation-a Lagrangian point of view," Communications in Partial Differential Equations, vol. 32, no. 10-12, pp. 1511-1549, 2007.

[14] H. Holden and X. Raynaud, "Dissipative solutions for the Camassa-Holm equation," Discrete and Continuous Dynamical Systems A, vol. 24, no. 4, pp. 1047-1112, 2009.

[15] Z. Xin and P. Zhang, "On the weak solutions to a shallow water equation," Communications on Pure and Applied Mathematics, vol. 53, no. 11, pp. 1411-1433, 2000.

[16] A. Constantin and W. A. Strauss, "Stability of a class of solitary waves in compressible elastic rods," Physics Letters A, vol. 270, no. 3-4, pp. 140-148, 2000.

[17] H. Holden and X. Raynaud, "Global conservative solutions of the generalized hyperelastic-rod wave equation," Journal of Differential Equations, vol. 233, no. 2, pp. 448-484, 2007.

[18] G. M. Coclite, H. Holden, and K. H. Karlsen, "Global weak solutions to a generalized hyperelastic-rod wave equation," SIAM Journal on Mathematical Analysis, vol. 37, no. 4, pp. 10441069, 2005.

[19] T. Kato, "Quasi-linear equations of evolution, with applications to partial differential equations," in Spectral Theory and Differential Equations, W. N. Everitt, Ed., vol. 448 of Lecture Notes in Mathematics, pp. 25-70, Springer, Berlin, Germany, 1975. 


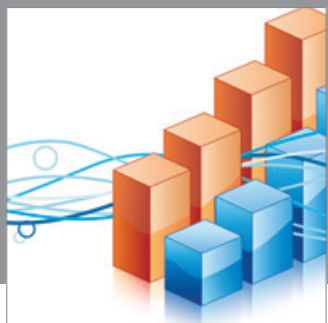

Advances in

Operations Research

mansans

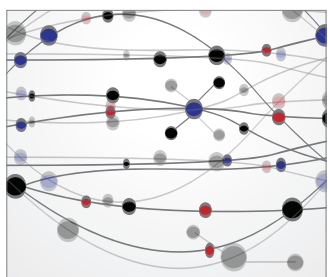

The Scientific World Journal
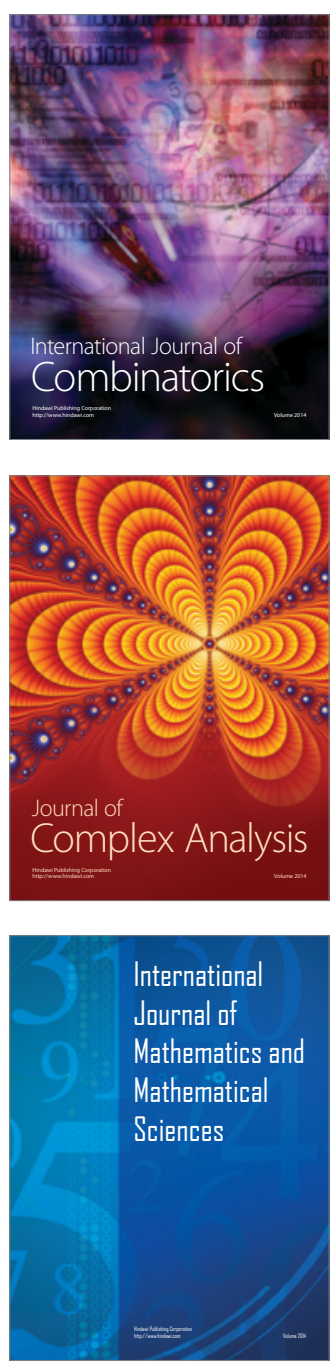
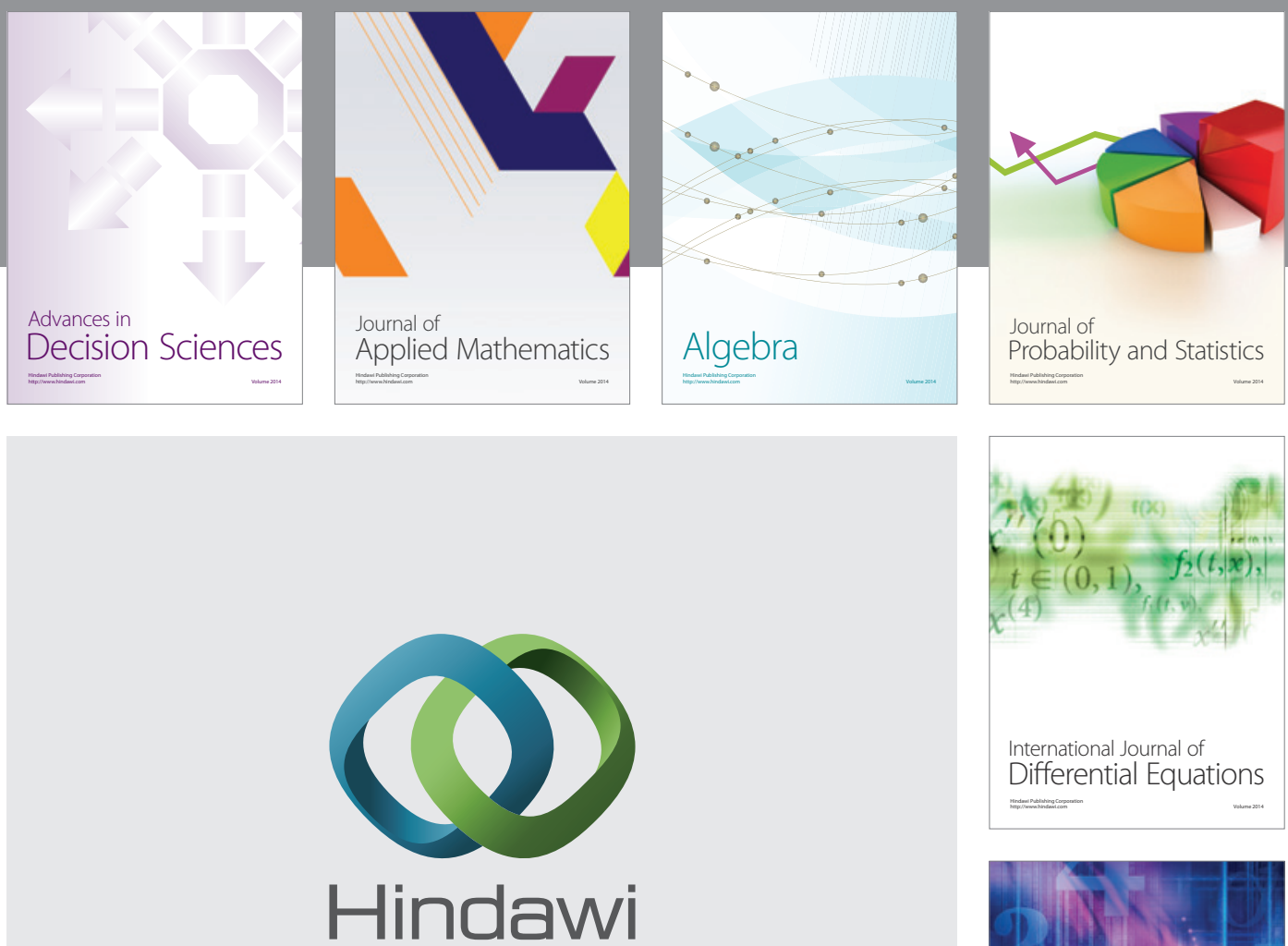

Submit your manuscripts at http://www.hindawi.com
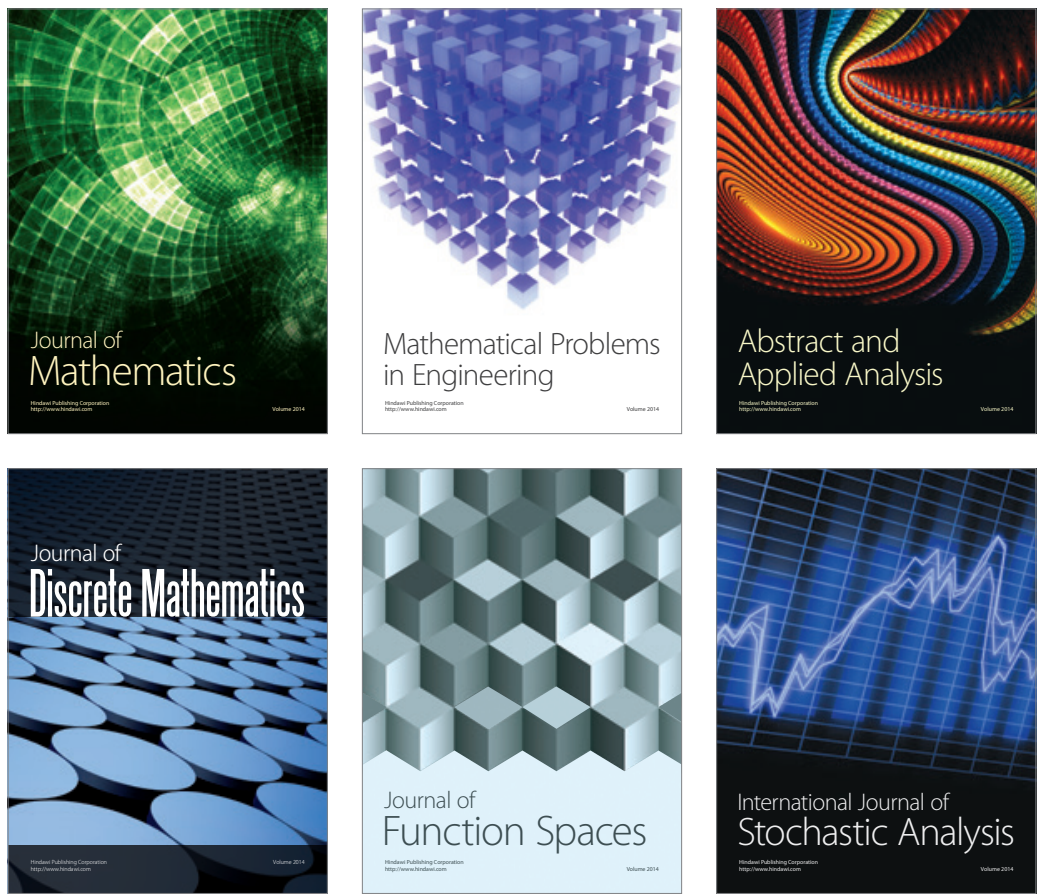

Journal of

Function Spaces

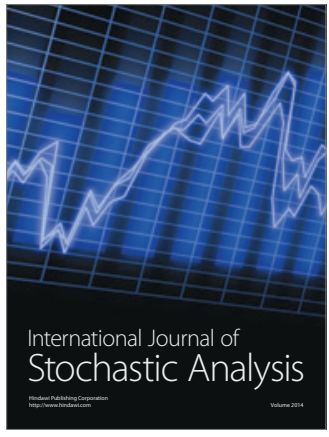

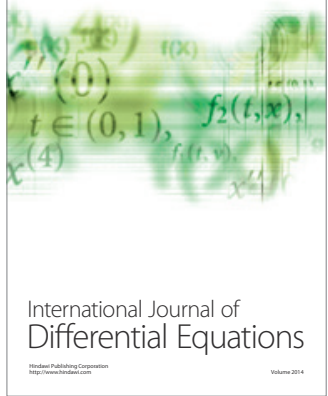
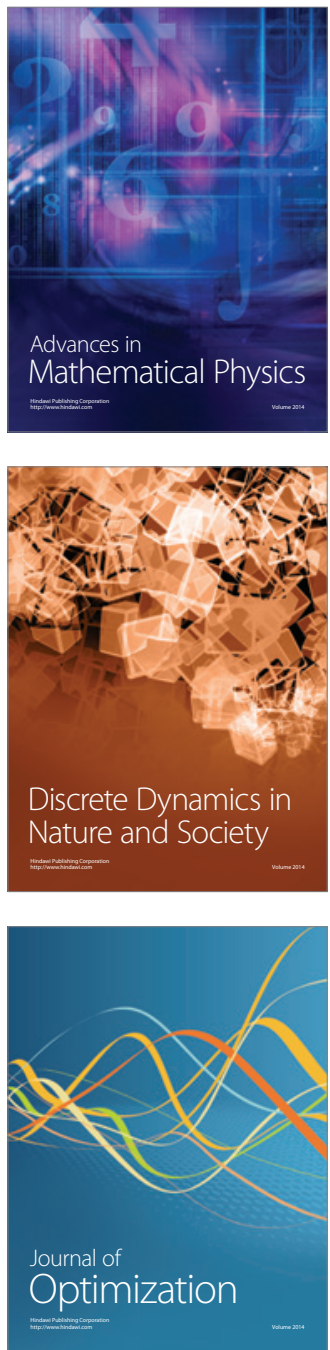